\title{
Magnetic resonance imaging in interventional therapy of patients with acute myocardial infarction prior to and after treatment
}

\author{
YUZHOU LI ${ }^{1}$, CHUNRONG LI $^{1}$, HONGRUI JIN ${ }^{2}$ and WENQI HUANG ${ }^{1}$ \\ ${ }^{1}$ Medical Image Center, The First People's Hospital of Shangqiu, Shangqiu, Henan 476100; \\ ${ }^{2}$ Department of Magnetic Resonance Imaging, The First Affiliated Hospital of Zhengzhou University, \\ Zhengzhou, Henan 450052, P.R. China
}

Received May 12, 2016; Accepted July 21, 2016

DOI: 10.3892/etm.2016.3537

\begin{abstract}
The aim of the study was to investigate the cardiac magnetic resonance (CMR) imaging in interventional therapy of patients with acute myocardial infarction prior to and after treatment. Fifty-six cases of AMI patients with elective treatment by percutaneous coronary intervention (PCI) were continuously selected. Patients with an incidence of 7-10 days were treated with CMR and echocardiography to evaluate the quality of myocardial infarction, visual score method (VSM), wall motion score abnormality, left ventricular end-diastolic diameter (LVEDD), left ventricular end-systolic diameter (LVESD) and left ventricular ejection fraction (LVEF). Patients with an incidence of 10-14 days were treated with PCI, and CMR and echocardiography were evaluated after 6 months, after which the occurrence of major adverse cardiac events (MACE) were compared. The infarction quality, VSM score and wall motion abnormality (WMA) score were significantly reduced following surgery, and the difference was statistically significant $(\mathrm{P}<0.05)$. Ultrasound evaluation of LVEDD, LVESD, and LVEF prior to and after surgery was compared, and the difference was not statistically significant $(\mathrm{P}>0.05)$. Evaluation of the magnetic resonance imaging (MRI) in LVEDD prior to surgery was increased compared with that of the ultrasound in LVEDD, whereas MRI in LVESD and LVEF was decreased compared to that of the ultrasound obtained for LVESD and LVEF. Additionally, postoperative LVEDD was
\end{abstract}

Correspondence to: Dr Hongrui Jin, Department of Magnetic Resonance Imaging, The First Affiliated Hospital of Zhengzhou University, 1 Jianshe East Road, Zhengzhou, Henan 450052, P.R. China

E-mail: jin_hongrui1@163.com

Key words: cardiac magnetic resonance imaging, acute myocardial infarction, interventional therapy, ultrasonic echocardiography, myocardial infarction quality, visual scoring method, wall motion abnormality score reduced compared with preoperative LVEDD, whereas LVEF was increased, and the difference was statistically significant $(\mathrm{P}<0.05)$. However, the evaluation of LVESD using the two methods exhibited no significant change. MACE occurred in $7(12.5 \%)$ of 56 cases. The infarction quality of patients in the MACE group following surgery indicated that VSM and WMA scores were significantly higher than the group without MACE, while LVEF was lower than the MACE group following surgery, and the difference was statistically significant $(\mathrm{P}<0.05)$, albeit the ultrasound results of LVEF indicated no difference. In conclusion, CMR evaluation of AMI patients with elective PCI treatment in myocardial remodeling and cardiac function were more sensitive and accurate than with cardiac ultrasound.

\section{Introduction}

Interventional therapy in acute myocardial infarction has been widely applied in the clinic. Objective and accurate assessment of the scope and extent of myocardial infarction, cardiac function and ventricular remodeling prior to and following surgery have become the focus of recent studies $(1,2)$. Conventional echocardiography and speckle tracking techniques have become the preferred examination method in clinical treatment as they are non-invasive, reproducible, and high in sensitivity and accuracy (3). However, the measurements of this method are not stable (4).

Cardiac magnetic resonance (CMR) imaging has high spatial resolution, and has a high recognition rate of myocardial infarction, degree of permeability, edema, bleeding, inflammation and other syndromes (5). In particular, delayed enhanced imaging (DE) was prepared to assess the scope and extent of myocardial infarction and impaired cardiac function, and post-processing software such as computer-aided volume methods and visual score method (VSM) were employed as optimal methods for quantitative detection of infracted myocardium (6,7).

The present study used CMR to assess myocardial infarction prior to and after AMI conducting percutaneous coronary intervention (PCI). The correlation between cardiac prognosis and echocardiography was compared and analyzed. 


\section{Materials and methods}

Subjects. Fifty-six cases of patients with AMI (incidence time of $>24$ h) were continuously selected at the FirstAffiliated Hospital of Zhengzhou University (Henan, China) from June, 2014 to June, 2015. There were indications of elective PCI, but no contraindications. Exclusion criteria for the study were: uncontrolled high blood pressure and diabetes, recent major operations and gastrointestinal bleeding history, malignant tumor, cerebral vascular diseases, coagulation dysfunction, severe insufficiency of liver and kidneys, contrast agent allergy, radiography failure, and high interventional risk of radiography assessment. Other exclusion factors were: magnetic resonance examination was not completed; patients exhibited cardiac shock, malignant arrhythmia, acute left heart failure and severe disease; less than one-year expected survival time; poor compliance; incomplete follow-up data and loss of follow-up.

The study obtained the approval of the Ethics Committee of the FirstAffiliated Hospital of Zhengzhou University and informed consent of the patients and their relatives. Detection with echocardiography and CMR was carried out in the incidence of 7-10 days to evaluate the myocardial infarction quality, VSM, wall motion abnormality (WMA) score, left ventricular end-diastolic diameter (LVEDD), left ventricular end-systolic diameter (LVESD), and left ventricular ejection fraction (LVEF). For the incidence of 10-14 days, PCI was employed, and detection with echocardiography and CMR were carried out again after 6 months, after which the incidence of major adverse cardiac events (MACE) was compared. The treatment of PCI was completed by the same surgical and nursing team, in line with standard medical procedures. There were 30 men and 26 women, aged 48-72 years, with an average age of $62.5 \pm 13.6$ years. The time of incidence was from 26 to $48 \mathrm{~h}$ with an average of $32.4 \pm 5.7 \mathrm{~h}$. Of the 56 cases, there were 21 cases of ST-segment elevation myocardial infarction (STEMI) and 35 cases of non-ST-elevation myocardial infarction (NSTEMI). In addition, there were 20 cases of anterior descending artery disease, 10 cases of circumflex artery, 20 cases of right coronary artery, and 6 cases of $\geq 2$ lesions. Each patient was implanted with 1-3 stents, with an average of $1.5 \pm 0.6 \mathrm{~mm}$. The length of each stent was $10-25 \mathrm{~mm}$, with and average of $15.7 \pm 4.6 \mathrm{~mm}$.

Detection method of magnetic resonance. A 3.0T superconductive magnetic resonance imager (Avanto; Siemens, Erlangen, Germany) was utilized, with a maximum gradient field of $45 \mathrm{mT} / \mathrm{m}$, maximum gradient slew rate of $200 \mathrm{mT} / \mathrm{m} \cdot \mathrm{msec}$. In addition, an 8 channel body surface coil and 6 channel spinal coil, wireless echocardiography vector template, with high-pressure syringe (Ulrich Medical, Berlin, Germany) were used. The gadopentetate dimeglumine of Schering AG (Berlin, Germany) was employed as the contrast agent. For conventional scanning, fast spin echo sequence was utilized to observe the morphology of the heart and large blood vessel, cardiovascular film was utilized for retrospective echocardiography gated to enter procession gradient echo sequence. Left ventricular two-chamber heart long axis, four-chamber heart long axis, left ventricular inflow and outflow tract, left ventricular outflow tract section, six-layer fractional movie, and cardiac function analysis were carried out using scanning and

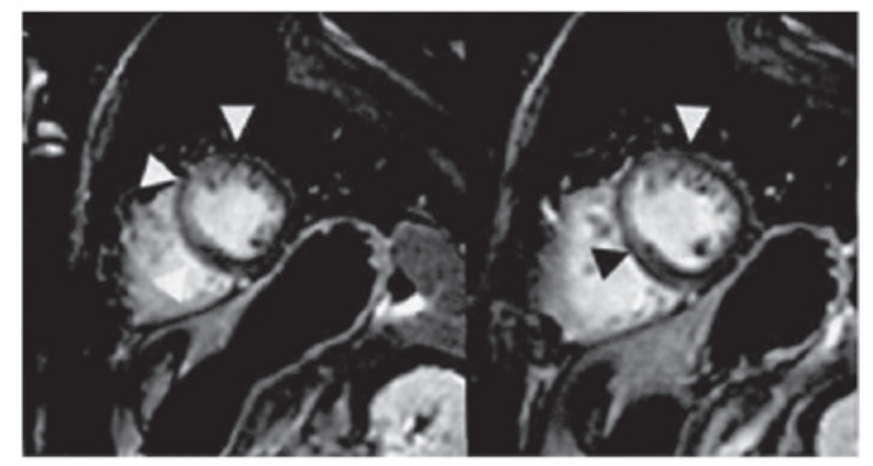

Figure 1. Magnetic resonance delayed enhancement schematic (display of the intensive shadow of apical, basal anterior segment, anterior septal and intermediate anterior segment delayed enhancement).

cardiovascular film, respectively. Argus 4D software (Siemens Healthcare, Eresing, Germany) was utilized to analyze the data. Multimodality workplace (MMWP) workstation was to measure LVEDD, LVESD, and LVED. The contrast agent enhanced the first-pass myocardial perfusion with a $4-5 \mathrm{ml} / \mathrm{sec}$ $0.1 \mathrm{mmol} / \mathrm{kg}$ flow rate, which was initiated at the same time as the scanning. TSENSE EPIGER sequence was used to conduct T1WI scanning (8), which constituted the contrast agent phase-sensitive with inversion recovery, including 6 layers of left ventricular short axis view, 1 layer of left ventricular two-chamber view, and four-chamber view. WMA involves semi-quantitative scoring in the sequence of grade 0-4 (0, normal; 1 , reduced movement; 2 , non-movement; 3 , contradictory movement; and 4 , the formation of ventricular aneurysm). Three standard short axes were selected (base, middle, apical) when the transmembrane extent with delayed enhancement was evaluated by VSM, and each segment was scored in accordance with the transmural extent: 0 , no enhancement; $1,0-25 \%$ enhancement; 2, 26-50\% enhancement; 3, 51-75\% enhancement; and 4, 76-100\% enhancement (Fig. 1). The scores of all the stages were added, yielding a total score of VSM. The result was independently analyzed by two experienced physicians. If the result was inconsistent, it would be analyzed by the third physician.

Echocardiography examination. Philips iE33 ultrasonic diagnostic apparatus and S5-1 probe (both from Philips Medical Systems, Inc., Bothell, WA, USA) with a frequency of 2-4 MHz were used to conduct M-type-sampling on the standard left ventricular short axis mitral valve under the guidance of the two-dimensional echocardiography, to ensure the sample line be vertical to the ventricular septal posterior wall, measuring LVEDD, LVESD, and automatically outputting LVED according to the Teichholz correction formula (9). The samples were measure three times and the average was taken.

MACE. MACE was defined as target vessel reconstruction, recurrence of angina and myocardial infarction, new heart failure and sudden cardiac death.

Statistical analysis. SPSS 19.0 software (SPSS Inc., Chicago, IL, USA) was used to input and analyze the data. Quantitative 
Table I. MRI evaluation in myocardial infarction.

\begin{tabular}{lccr}
\hline & $\begin{array}{c}\text { Infarction } \\
\text { quality (g) }\end{array}$ & $\begin{array}{l}\text { VSM } \\
\text { score }\end{array}$ & $\begin{array}{l}\text { WMA } \\
\text { score }\end{array}$ \\
\hline Preoperative & $8.3 \pm 1.2$ & $11.2 \pm 1.5$ & $6.2 \pm 0.8$ \\
Postoperative & $6.9 \pm 1.0$ & $7.6 \pm 1.2$ & $3.7 \pm 0.5$ \\
t & 6.302 & 6.754 & 7.221 \\
P-value & 0.037 & 0.028 & 0.013 \\
\hline
\end{tabular}

MRI, magnetic resonance imaging; VSM, visual score method; WMA, wall motion abnormality.

data are presented as mean \pm standard deviation. Comparisons among groups were tested using the independent sample t-test, and the intergroup comparison was tested using the paired t-test. Qualitative data are expressed as the number of case or percentage, and comparisons among groups were tested using the $\chi^{2}$ test. $\mathrm{P}<0.05$ was considered to indicate a statistically significant difference.

\section{Results}

Magnetic resonance imaging (MRI) evaluation in myocardial infarction. Postoperative infarction quality (6.9 \pm 1.0 from $8.3 \pm 1.2)$, and VSM and WMA scores (7.6 \pm 1.2 and 3.7 \pm 0.5 , respectively) were significantly reduced. The difference was statistically significant $(\mathrm{P}<0.05)$ (Table I).
Ultrasound and magnetic resonance evaluation in cardiac function. The comparison of ultrasound evaluation in LVEDD, LVESD, LVEF before and after the operation indicated the difference was of no statistical significance $(\mathrm{P}>0.05)$. LVEDD was increased following evaluation by magnetic resonance prior to surgery as compared to that by ultrasound, whereas LVESD and LVEF were reduced. Additionally, postoperative LVEDD was reduced compared to that prior to surgery, while LVEF was increased, and the difference was statistically significant $(\mathrm{P}<0.05)$. However, no significant change was found in LVEDD (Table II).

Correlation of the two evaluation methods and MACE. MACE occurred in 7 (12.5\%) of 56 cases. The infarction quality, and VSM and WMA scores of patients in the MACE group were significantly higher than the group without MACE. Post-operative LVED was lower than the group without MACE, and the difference was statistically significant $(\mathrm{P}<0.05)$. There was no difference in the evaluation of ultrasound (Table III).

\section{Discussion}

Effective and accurate measurement of myocardial infarction quality is of great value in evaluating the prognosis of patients. Animal experiments have shown that infarction quality measured by delayed and enhanced MRI is highly conformed with the quality of the scar displayed by TTC staining, which is considered to be the gold standard for the evaluation of myocardial necrosis on histology $(10,11)$. Cardiac MRI evaluation of myocardial viability quality and detection of infarction

Table II. Ultrasound and magnetic resonance evaluation in cardiac function.

\begin{tabular}{|c|c|c|c|c|c|c|}
\hline & \multicolumn{2}{|c|}{ LVEDD (mm) } & \multicolumn{2}{|c|}{ LVESD (mm) } & \multicolumn{2}{|c|}{ LVEF (\%) } \\
\hline & Preoperative & Postoperative & Preoperative & Postoperative & Preoperative & Postoperative \\
\hline Ultrasound & $54.6 \pm 1.2$ & $54.5 \pm 1.5$ & $27.5 \pm 2.2$ & $27.3 \pm 2.0$ & $49.6 \pm 2.4$ & $51.2 \pm 3.0$ \\
\hline MRI & $56.4 \pm 1.3$ & $55.7 \pm 1.2$ & $25.6 \pm 2.0$ & $25.4 \pm 2.1$ & $45.7 \pm 2.3$ & $49.7 \pm 3.3$ \\
\hline $\mathrm{t}$ & 5.324 & 5.124 & 5.629 & 5.748 & 6.345 & 6.528 \\
\hline P-value & 0.039 & 0.040 & 0.035 & 0.034 & 0.032 & 0.030 \\
\hline
\end{tabular}

LVEDD, left ventricular end-diastolic diameter; LVESD, left ventricular end-systolic diameter; LVEF, left ventricular ejection fraction; MRI, magnetic resonance imaging.

Table III. The correlation of the two evaluation methods and MACE.

\begin{tabular}{lccccc}
\hline Groups & Infarction quality $(\mathrm{g})$ & VSM score & WMA score & LVEF (MRI) & LVEF (ultrasound) \\
\hline Group with MACE & $7.4 \pm 1.6$ & $7.9 \pm 1.6$ & $4.1 \pm 0.6$ & $46.5 \pm 3.5$ & $51.0 \pm 3.6$ \\
Group without MACE & $6.5 \pm 1.3$ & $7.3 \pm 1.4$ & $3.4 \pm 0.3$ & $51.2 \pm 3.2$ & $51.4 \pm 3.5$ \\
t & 6.328 & 6.120 & 6.635 & 7.203 & 0.639 \\
P-value & 0.034 & 0.036 & 0.030 & 0.018 & 0.548
\end{tabular}

MACE, major adverse cardiac events; VSM, visual score method; WMA, wall motion abnormality; LVEF, left ventricular ejection fraction; MRI, magnetic resonance imaging. 
quality are based on systolic dysfunction and myocardial perfusion defects. However, the presence of cell metabolism, survival of cell membrane integrity, and potentially contractile reserve are useful in the enhancement contractile response to positive inotropic agents (12). The basic principle of delayed MRI is the use of paramagnetic contrast agents to reduce the myocardial $\mathrm{T} 1$ relaxation time from rapidly entering the vascular bed, and distributing in the extravascular space (13). In addition, the aim of MRI is to evaluate myocardial infarction size, quality, and myocardial injury degree through the dynamic process of cardiac chamber and myocardium (13). The strength of myocardial tissue signal depends on the blood flow volume, tissue perfusion, size of the extracellular space, and the distribution of the contrast agent in the myocardium (14). A high signal area of delayed enhancement is an irreversible necrotic myocardium. The main reason for myocardial infarction is structural damage of myocardial cells and microvascular damage, and the mechanism underlying the infarction area may be the delay of non-active tissue contrast agents clustered at the entry and exit points, and the distribution volume of the contrast agent in the active and inactive regions (15).

Recent findings show that revascularization is not affected by MRI on the first day following stent implantation, and no stent artifacts are produced, which can accurately determine the quality of myocardial infarction, the degree of permeability, the degree of ventricular wall motion, and left ventricular function (16). Thus, the infarction quality, and VSM and WMA socres were significantly reduced, with the difference being statistically significant. In the present study, the difference was of no statistical significance in the comparison of ultrasound evaluation in LVEDD, LVESD, and LVEF prior to and following surgery. LVEDD was increased by the evaluation of magnetic resonance imaging prior to surgery compared to that by ultrasound, whereas LVESD and LVEF were reduced. Additionally, postoperative LVEDD was reduced compared to that prior to surgery, whereas LVEF was increased, with the difference being statistically significant, albeit no significant change was found in LVEDD. Magnetic resonance measurement is generally lower than that for ultrasound, considering that the shape of the ventricular cavity may be irregular following myocardial infarction, which makes left ventricular volume or inner measurement prone to bias under echocardiography (17). In addition, over-reliance on ultrasound techniques and experience of the operator may cause endocardial inappropriate depiction and is subjected to the limitation of the acoustic window, adding difficulty in distinguishing the myocardial, endocardial, and epicardial adipose layer, resulting in the measured value being extremely large (17). However, CMR is an objective, quantitative indication that does not assume the geometry of the left ventricle, especially for existing ventricular remodeling and expansion of the heart chamber to obtain better ejection fraction, and has strong repeatability and good consistency (18). The infarction quality, and VSM and WMA scores of the patients in the MACE group were significantly higher than the group without MACE. Postoperative LVEF was lower than the group without MACE, and the difference was statistically significant, whereas there was no difference found in the ultrasound evaluation of LVEF. The results suggest that CMR measurement of myocardial infarction and the changes in cardiac function are more sensitive, which is associated with prognosis.

In conclusion, CMR evaluation of AMI with elective PCI treatment in myocardial infarction remodeling and cardiac function is more sensitive and accurate compared to echocardiography. However, further investigations are required to confirm the above results.

\section{References}

1. Elmariah S, Smith SJ and Fuster V: Late medical versus interventional therapy for stable ST-segment elevation myocardial infarction. Nat Clin Pract Cardiovasc Med 5: 42-52, 2008.

2. Degeare VS, Dangas G, Stone GW and Grines CL: Interventional procedures in acute myocardial infarction. Am Heart J 141: 15-24, 2001.

3. Woo JS, Yu TK, Kim WS, Kim KS and Kim W: Early prediction of myocardial viability after acute myocardial infarction by two-dimensional speckle tracking imaging. J Geriatr Cardiol 12: 474-481, 2015.

4. Jankauskienė E, Orda P, Rumbinaitè E, Žaliaduonytė-Pekšienè D, Steponavičiutè R, Krasauskienè A, Vaškelytė JJ and Bunevičius R: Left ventricular function by speckle-tracking echocardiography in patients with low-T3 syndrome and acute myocardial infarction. Medicina (Kaunas) 51: 209-216, 2015.

5. Stuckey DJ, Ishii H, Chen QZ, Boccaccini AR, Hansen U, Carr CA, Roether JA, Jawad H, Tyler DJ, Ali NN, Clarke K and Harding SE: Magnetic resonance imaging evaluation of remodeling by cardiac elastomeric tissue scaffold biomaterials in a rat model of myocardial infarction. Tissue Eng Part A 16: 3395-3402, 2010.

6. Tsadok Y, Friedman Z, Haluska BA, Hoffmann R and Adam D: Myocardial strain assessment by cine cardiac magnetic resonance imaging using non-rigid registration. Magn Reson Imaging 34: 381-390, 2016.

7. Romero J, Lupercio F, Díaz JC, Goodman-Meza D, Haramati LB, Levsky JM, Shaban N, Piña I and Garcia MJ: Microvascular obstruction detected by cardiac MRI after AMI for the prediction of LV remodeling and MACE: a meta-analysis of prospective trials. Int J Cardiol 202: 344-348, 2016.

8. Yamamuro M, Tadamura E, Kanao S, Okayama S, Okamoto J, Urayama S, Kimura T, Komeda M, Kita T and Togashi K: Cardiac functional analysis by free-breath real-time cine CMR with a spatiotemporal filtering method, TSENSE: Comparison with breath-hold cine CMR. J Cardiovasc Magn Reson 8: 801-807, 2006.

9. Scopinaro F, Banci M, Vania A, Tavolaro R, Schillaci O, Tisei A, Werner B, Digilio G, Ventriglia F and Colloridi V: Radioisotope assessment of heart damage in hypertransfused thalassaemic patients. Eur J Nucl Med 20: 603-608, 1993.

10. Suhail MS, Wilson MW, Hetts SW and Saeed M: Magnetic resonance imaging characterization of circumferential and longitudinal strain under various coronary interventions in swine. World J Radiol 5: 472-483, 2013.

11. Haase J, Bayar R, Hackenbroch M, Storger H, Hofmann M, Schwarz CE, Reinemer H, Schwarz F, Ruef J, Sommer T: Relationship between size of myocardial infarctions assessed by delayed contrast-enhanced MRI after primary PCI, biochemical markers, and time to intervention. J Interv Cardiol 17: 367-373, 2004.

12. Saeed M, Van TA, Krug R, Hetts SW and Wilson MW: Cardiac MR imaging: current status and future direction. Cardiovasc Diagn Ther 5: 290-310, 2015.

13. Park CH, Choi EY, Yoon YW, Kwon HM, Hong BK, Lee BK, Min PK, Greiser A, Paek MY, Hwang SH, et al: Quantitative T2 mapping after reperfusion therapy in patients with acute myocardial infarction: a comparison with late gadolinium enhancement and cine MR imaging. Magn Reson Imaging 33: 1246-1252, 2015.

14. Ma N, Cheng H, Lu M, Liu Q, Chen X, Yin G, Zhu H, Zhang L, Meng X, Tang Y, et al: Magnetic resonance imaging with superparamagnetic iron oxide fails to track the long-term fate of mesenchymal stem cells transplanted into heart. Sci Rep 5: 9058, 2015. 
15. Bönner F, Merx MW, Klingel K, Begovatz P, Flögel U, Sager M, Temme S, Jacoby C, Salehi Ravesh M, Grapentin C, et al: Monocyte imaging after myocardial infarction with 19F MRI at $3 \mathrm{~T}$ : a pilot study in explanted porcine hearts. Eur Heart $\mathrm{J}$ Cardiovase Imaging 16: 612-620, 2015.

16. Rischpler C, Langwieser N, Souvatzoglou M, Batrice A, van Marwick S, Snajberk J, Ibrahim T, Laugwitz KL, Nekolla SG and Schwaiger M: PET/MRI early after myocardial infarction: evaluation of viability with late gadolinium enhancement transmurality vs. 18F-FDG uptake. Eur Heart J Cardiovasc Imaging 16: 661-669, 2015.
17. No authors listed: Recommendations for cardiac chamber quantification by echocardiography in adults: an update from the American Society of Echocardiography and the European Association of, Cardiovascular Imaging. Eur Heart J Cardiovasc Imaging 17: 412, 2016.

18. Kidambi A, Biglands JD, Higgins DM, Ripley DP, Zaman A, Broadbent DA, McDiarmid AK, Swoboda PP, Al Musa T, Erhayiem B, et al: Susceptibility-weighted cardiovascular magnetic resonance in comparison to T2 and T2 star imaging for detection of intramyocardial hemorrhage following acute myocardial infarction at 3 Tesla. J Cardiovasc Magn Reson 16: 86, 2014. 